\title{
Investigational pharmacology for low back pain
}

This article was published in the following Dove Press journal:

Journal Pain Research

4 September 2010

Number of times this article has been viewed

\author{
Avinash K Bhandary' \\ Gary P Chimes ${ }^{2}$ \\ Gerard A Malanga ${ }^{3}$ \\ 'Department of Physical Medicine \\ and Rehabilitation, ${ }^{2}$ Department of \\ Physical Medicine and Rehabilitation, \\ University of Pittsburgh Medical \\ Center, Pittsburgh, PA, USA; ${ }^{3} \mathrm{New}$ \\ Jersey Sports Medicine Institute; \\ Overlook Hospital; Mountainside \\ Hospital; Rehabilitation Medicine \\ and Electrodiagnosis, St Michael's \\ Medical Center; Horizon Healthcare \\ Worker's Compensation Services, \\ Blue Cross and Blue Shield Worker's \\ Compensation, Summit, NJ, USA
}

Study design: Review and reinterpretation of existing literature.

Objective: This review article summarizes the anatomy and pathogenesis of disease processes that contribute to low back pain, and discusses key issues in existing therapies for chronic low back pain. The article also explains the scientific rationale for investigational pharmacology and highlights emerging compounds in late development.

Results/conclusion: While the diverse and complex nature of chronic low back pain continues to challenge clinicians, a growing understanding of chronic low back pain on a cellular level has refined our approach to managing chronic low back pain with pharmacology. Many emerging therapies with improved safety profiles are currently in the research pipeline and will contribute to a multimodal therapeutic algorithm in the near future. With the heterogeneity of the patient population suffering from chronic low back pain, the clinical challenge will be accurately stratifying the optimal pharmacologic approach for each patient.

Keywords: low back pain, investigational, pharmacology, drugs

\section{Introduction}

Low back pain (LBP) has reached epidemic proportions in Western societies with reported lifetime prevalence rates more than $70 \% .{ }^{1}$ LBP is a musculoskeletal symptom defined as discomfort in the lumbosacral region of the back. The discomfort may or may not radiate to the legs, hips, and buttocks. LBP is considered acute if the discomfort persists 6 weeks or less and is considered chronic if persists longer than 12 weeks. While the population of patients suffering from LBP continues to expand, the clinical challenges of effectively treating back pain persist. This reflects the multidimensional nature of LBP and heterogeneity of the population it affects. In the past decade, emerging treatments and preventive approaches of care for conditions such as respiratory and circulatory diseases have been successful in reducing the cost of social security disability. This contrasts with LBP, where the population of disabilities beneficiaries continues to expand despite an array of therapeutic measures. ${ }^{2}$

Despite a substantial expansion in other therapeutic approaches, pharmacotherapy remains central to the management of LBP. Analysis of the United States national expenditures for back pain finds that the costs allocated to prescription medications have increased at a rate greater than any other service category, which includes imaging, emergency department visits, and inpatient and outpatient services. ${ }^{3}$

Fortunately, there are promising emerging treatments that may help in optimizing pharmacologic management. The focus of LBP pharmacology has shifted from
Correspondence: Gary P Chimes Department of Physical Medicine and Rehabilitation, University of Pittsburgh Medical Center, Pittsburgh, PA, USA

$\mathrm{Tel}+\mathrm{I} 4128580337$

Fax + | $412372 \mid 493$

Email garypchimes@gmail.com 
symptom-directed management to a better understanding of pain mechanisms on a basic science level. Disease modification, molecular based pharmacologic targets, and biomarkers of disease processes are all new and exciting pathways for investigational pharmacology.

Investigational pharmacology for LBP is a topic of increasing significance on clinical and fiscal levels. This article will look at common etiologies of back pain, review emerging treatments along the research pipeline (Figure 1), their scientific rationale, and the promise of their therapeutic possibilities.

\section{Osteoarthritis-induced low back pain}

LBP affects $80 \%$ of the world's population. The common cold is the only disorder that occurs more frequently than LBP. $^{4}$ The lumbar spine is a common site for symptomatic pain associated with osteoarthritis (OA), because it is a primary weight-bearing structure for most functional activities. Risk factors predisposing to osteoarthritis-induced LBP range from race and gender to biomechanics and age. Pharmacologic treatment options for OA are diverse both in terms of mechanisms of action and delivery formulations. However, no single agent has been demonstrated to consistently offer both a high level of tolerability and sustained degree of efficacy across a broad OA patient population. ${ }^{5}$

Patient satisfaction is shifting to medications that provide an improved long-term prognosis rather than short-term symptomatic relief. This has allowed pharmacotherapeutic strategies to focus on the biology of osteoarthritis on a molecular level in hopes to modify the disease and provide long- term results with improved safety profiles. Scientific rationale behind emerging pharmacology centers on the biomarkers of OA and will attempt to modify these inflammatory mediators. The relationship between inflammation and angiogenesis and prevalence of synovositis in OA also provides clinical ground for experimental strategies.

\section{Anatomy}

Aside from the complex psychological component of pain perception and referred pain, the ambiguous nature of LBP is underpinned by the numerous potential pain contributors within the anatomy. Clinicians must travel a path of exclusion, investigating the nature of disease processes, integrity, and the biomechanics of spinal anatomy before deciding upon the appropriate therapeutic regimen. Anatomic areas of interest include intervertebral discs, lumbar spinal nerves, sacroiliac and zygapophyseal joints (ZJ), muscles, ligaments, and tendons.

To locate the primary pain generator in LBP, physicians must correlate clinical findings with the appropriate pathophysiologic process. A crucial step in understanding the pathophysiology of LBP was made with the description of the degenerative cascade by Dr William Kirkaldy-Willis. The degenerative process that transforms an anatomically succinct lumbar spine into a source of pain was described by Kirkaldy-Willis in a 3-phase process. The first phase, termed the dysfunction phase, described the low back in terms of functional units. Each functional unit is composed of a 3 -joint complex consisting of an intervertebral disk and 2 ZJs. The dysfunction phase described the clinical presentation and pain produced by dysfunctionality of the 3 -joint complex. ${ }^{6}$

\begin{tabular}{|c|c|}
\hline Drug class & Drug \\
\hline 1. COX/LOX inhibitor & 1. Licofelone (ML3000) \\
\hline 2. COX-inhibiting nitric oxide donator (CINOD) & 2. Naproxcinod (AZD-3582) \\
\hline 3. COX-2 inhibitor & 3. Lumiracoxib \\
\hline 4. Delta opioid receptor agonist & 4. ADL5859, ADL5747 \\
\hline 5. $\mu$-opioid receptor agonist & 5. Loperamide \\
\hline 6. Antiresorptive pharmacology & 6. Salmon calcitonin \\
\hline 7. Neurotoxin & 7. Botulinum toxin \\
\hline 8. Ion channel (voltage and ligand gated) blocker & 8. Lacosamide \\
\hline 9. N-type calcium channel blocker & 9. Ziconotide, leconotide (CNSB004) \\
\hline 10. TRPV1-agonis & 10. Transacin (Transdolor, NGX-4010) \\
\hline 11. Cannabinoids & 11. Sativex \\
\hline 12. Antidepressants & 12. Venlafaxine \\
\hline
\end{tabular}

Figure I Pharmacology reviewed. 
The second stage, known as the unstable phase, described the pain generated from continuing stresses secondary to dysfunction of the 3-joint complex during movement. The third phase, termed the stabilization phase, referred to pathologic changes in the lumbar spine that produce stiffness, immobility, and eventual autofusion of the spine. ${ }^{6}$ These pathologic changes include fibrosis of the 3-joint complex, osteophyte production and subsequent central spinal canal, lateral recess and neural foramen narrowing, which lead to the production radicular leg pain.

With the responsibility of truncal load dissipation, torsion, flexion, and extension the interplay between lower back anatomic structures is significant. Abnormalities in pathoanatomic structures, such as muscle, tendon, ligament, or the sacroiliac joint produce poor postural control and altered kinematics that facilitate LBP. Two of the primary anatomical components of mechanical LBP are the intervertebral disc and ZJ.

The essential components of disc-mediated pain include internal disc disruption (IDD), which is the most common, disc torsion, and infection of the intervertebral disc known as diskitis. Discogenic pain is mediated through the mixed autonomic and somatic fibers of the sinuvertebral nerve. The sinuvertebral nerve innervates the posterior annulus and adhering longitudinal ligament and normally penetrates the outer $1-3 \mathrm{~mm}$ of the annulus or outer third of the disk. The annulus is significant in that it is responsible for the highest stress concentration during mechanical loading.

The ZJs, also commonly referred to as facet joints, are one of the most common sources of LBP. ${ }^{7}$ The ZJs are a pair of joints in the posterior aspect of the spine that bridge between the superior articular process of one vertebra and the inferior articular process of the vertebra immediately superior (Figure 2). The facets on the articular processes are covered by articular cartilage and a $1 \mathrm{~mm}$ thick fibrous capsule lined by synovial membrane, making this joint a true synovial joint. The function of the lumbar ZJ includes repetitive load transmission, stabilization of flexion and extension, and limiting axial rotation. Potential pain generators are interwoven throughout the complex neuroanatomy of the ZJ. Terminal branches of unmyelinated (Group 4) and thinly myelinated (Group 3) fibers are located throughout the synovium and periosteum. Nociceptors are found surrounding blood vessels near the synovium cells. In addition, the capsule of the $\mathrm{ZJ}$ is richly innervated with nociceptive and autonomic nerve fibers. ${ }^{8}$

Innervation of the $\mathrm{ZJ}$ begins with the spinal nerve at each lumbar spinal level, which divides into a ventral and dorsal

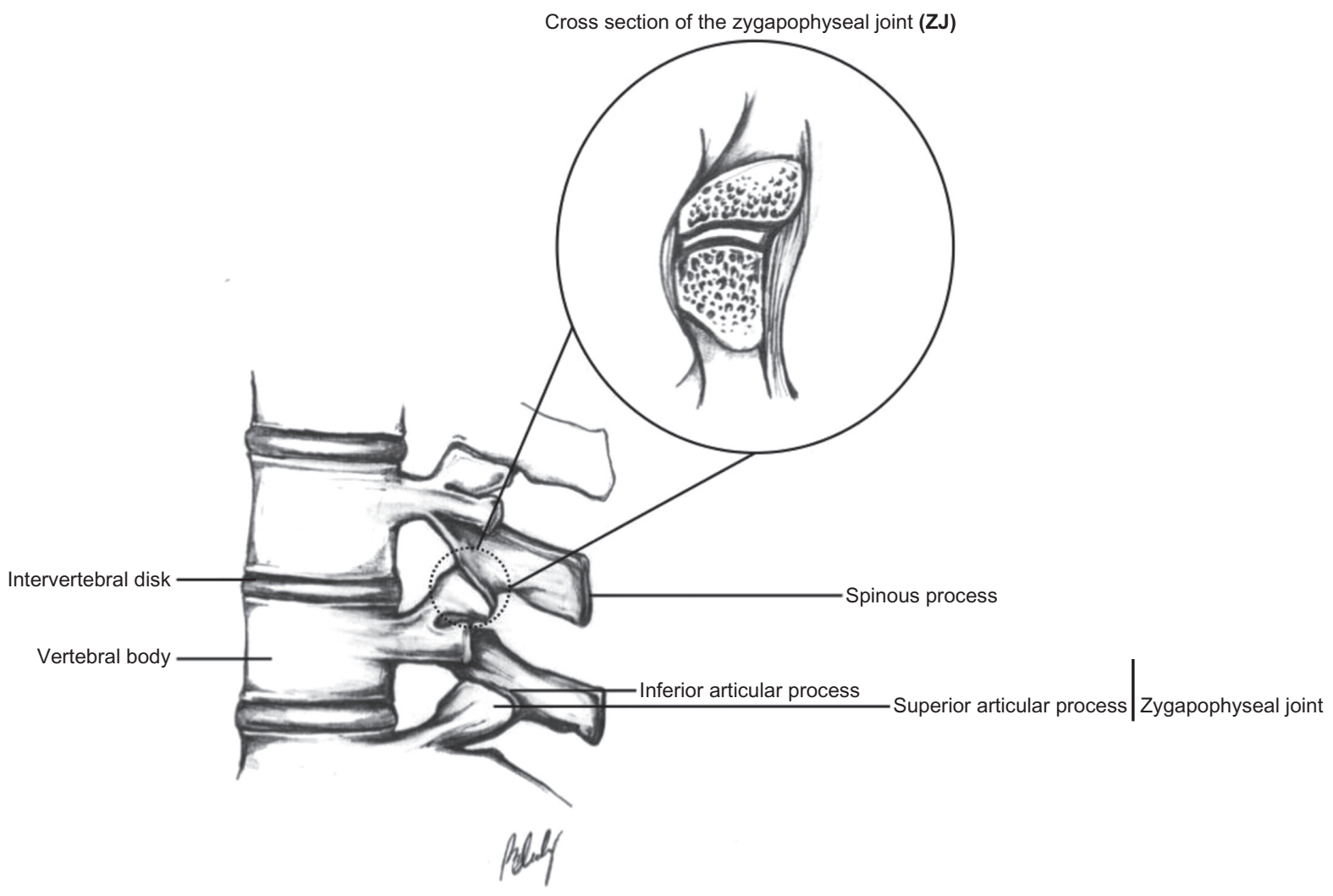

Figure 2 Zygapophyseal joint. 
primary ramus. The dorsal ramus divides into 3 branches, and the most medial branch supplies sensory fibers to 2 facet joint levels. The sensory fibers supply the inferior portion of a posterior facet and the superior part of the joint capsule at the next lower level. Therefore, each ZJ is supplied by sensory nerves from spinal nerves from 2 different segments. ${ }^{9}$ The perception of pain originates from inflammation and resultant sensitization of unmyelinated sensory nerves present in the osteoarthritic joint. This network of sensory nerve innervation to the ZJ is complex and often overlaps, making lumbar spinal pain difficult to localize.

\section{Pathogenesis}

Osteoarthritis-induced LBP represents step-wise structural failure of the lumbar spine that ultimately leads to an ingrowth of nerves and blood vessels. Lumbar spinal pain related to OA of the $\mathrm{ZJ}$ is closely related to disk height for several reasons. The height of the annulus determines the separation between the neural arches of adjacent vertebrae, and annulus collapse/ bulging in old discs can lead to more than $50 \%$ of the compressive force being resisted by the neural arch. ${ }^{10}$ With age and repetitive compressive loading, the trabecular network of the vertebral endplate sustains microdamage and allows for decompression of the adjacent nucleus, which subsequently transfers truncal load to the annulus. An irregular distribution of mechanical load throughout the disk ultimately shifts forces posteriorly to the ZJ and the ligmamentum flavum. The net result is that the posterior elements, particularly the ZJ, will bear a greater load, which contributes to further degeneration of the spine.

On a cellular level, disc degeneration occurs when an imbalance exists between the efforts of chondrocytes, the production of cartilaginous anabolic factors, and production of catabolic enzymes. Catabolic mediators include matrix metalloproteinases (MMP-3, MMP-13), inducible nitric oxide synthase (iNOS), interleukin-1-beta (IL-1B), and tumor necrosis factor (TNF-a). ${ }^{11}$ The catabolic pathways are induced by compressive forces, which also cause tissue hypoxia and bony matrix degeneration both of which are potent mediators of angiogenesis.

Angiogenesis is the proliferation of new blood vessels from preexisting blood vessels. Degeneration leads to inflammation, which is mediated by macrophages that produce inflammatory mediators as well as produce factors that initiate the angiogenic cascade. The association between osteochondral angiogenesis and pain behavior may be explained by perivascular nerve growth or stimulation of subchondral nerves following loss of osteochondral integrity. ${ }^{12}$
Angiogenesis perpetuates inflammation by providing permeable channels for leukotreine ingress which secrete proinflammatory factors. The sprouting network of unmyelinated nerve growth or neo-innervation that follows angiogenesis is a crucial element in the generation of pain. Angiogenesis may introduce sensory nerves into the aneural cartilage, and inflammation can sensitize nerves present in the joint. ${ }^{13}$ Therefore, a 'normally' insensate structure potentially becomes a candidate for pain in OA. This may also accelerate disease status via localized neurogenic inflammation. ${ }^{14}$

The symbiotic relationship between angiogenesis and inflammation occurs within the synovial membrane and is significant to the pathophysiology of OA. Increasing attention is being devoted to the contribution of synovositis in the pathology of OA. Synovositis is the result of an over expression of proinflammatory cytokines, such as IL-1B, and TNF, that drive degenerative catabolic activity. Thrombin is another protein in the synovial fluid which is a potential biomarker of synovial inflammation and is correlated with angiogenic factor VEGF expression. ${ }^{15}$ Synovositis has been identified in early and end-stage OA. Synovositis, therefore, although not a prerequisite for OA, may lead to a poor clinical outcome. ${ }^{13}$ Novel therapeutic interventions aiming to inhibit synovositis in OA may not only improve short-term symptoms but also reduce pain and disability in the long term.

\section{Prostanoids and receptors}

The development and severity of OA correlates with an increasing expression of cartilage-degrading enzymes, prostaglandins (PGs) and leukotrienes (LTs) in synovial fluid. The action of cyclooxygenase (COX) and 5-lipooxygenase (5-LOX) on arachidonic acid (AA) produces prostanoid enzyme products PGs and LTs. The COX enzyme exists in 3 isoforms COX-1, COX-2, and COX-3. Non-steroidal anti-inflammatory drugs (NSAIDs) pharmacologically inhibit both COX-1 and COX-2, producing substantial analgesia. The dual inhibition of NSAIDs prohibits the protective effect of PGs on gastric mucosa and leads to ulceration of the mucosa. Selective COX-2 inhibitors reduce PG formation, producing analgesia and inflammation relief. However, selective COX-2 inhibitors also have adverse effects on the cardiovascular (CV) system. The COX-3 isoform is a variant of COX-1 and has low enzymatic capability; its distribution and low abundance in the central nervous system and in periphery do not make this a compelling target for analgesia. ${ }^{14}$ Inhibition of both COX-1 and COX-2 shifts AA metabolism towards the 5-LOX pathway, which leads to subsequent LT production (Figure 3 ). 


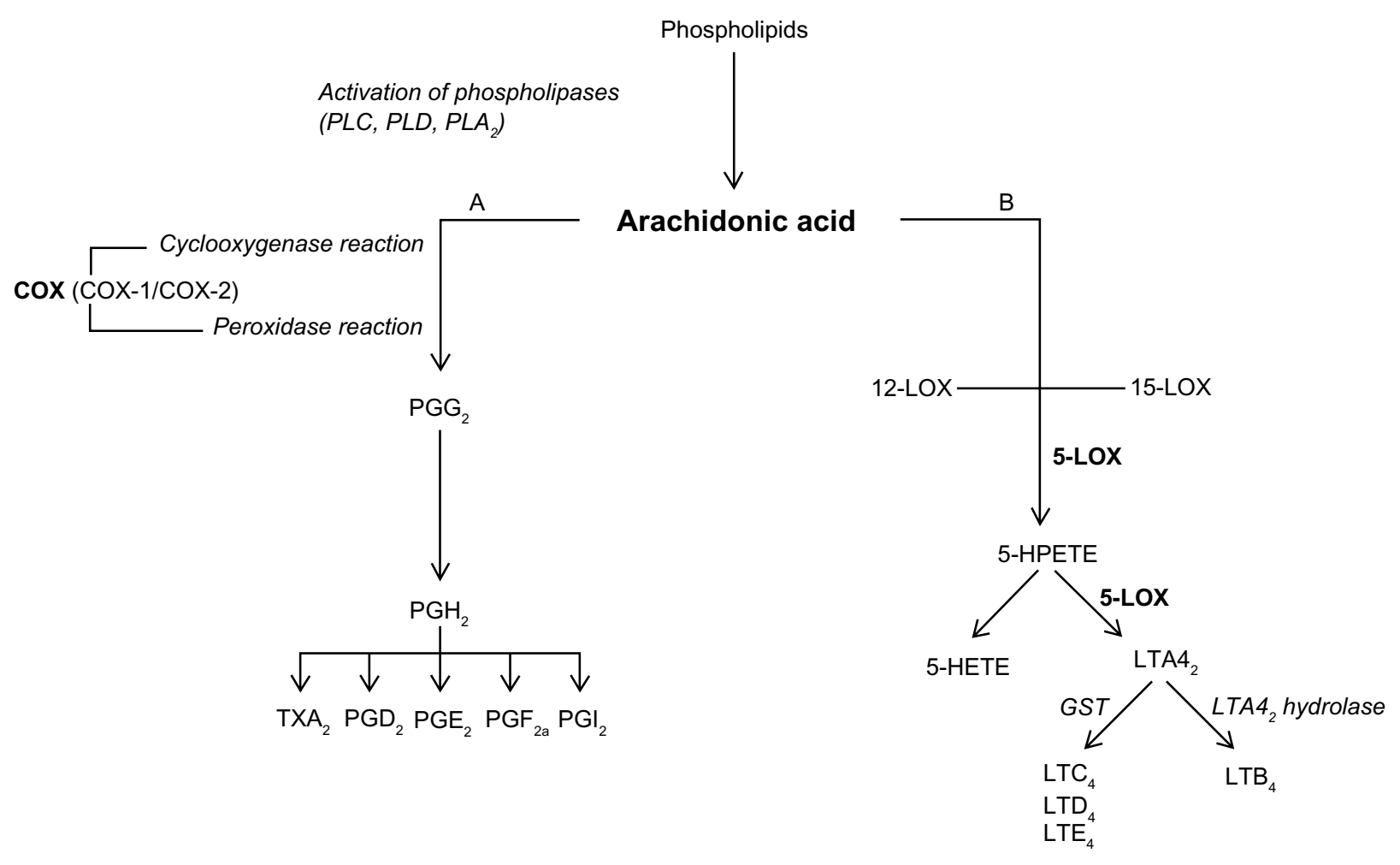

Figure 3 The cyclooxygenase (COX) pathway.

LTs are responsible for changes in vascular permeability that occur during acute inflammation. ${ }^{16}$ Therefore, LTs have been implied as contributing factors in NSAID induced gastropathy and are identified an inflammatory mediators along with PGs. The combination of 5-LOX inhibitors or LT receptor antagonists with NSAIDs has been reported to be beneficial not only in relieving pain and inflammation, but also in preventing or reducing NSAID induced gastric damage. ${ }^{17}$ Thus, the concept of dual inhibition (ie, COX and 5-LOX inhibition) has emerged as an alternative safe therapy for enhanced analgesic and anti-inflammatory effect with little or no gastric mucosal damage. ${ }^{18}$ Recently, dual inhibition was suggested to curtail the adverse $\mathrm{CV}$ effects otherwise reported with COX inhibition. ${ }^{19}$

\section{Prostanoid related pharmacology}

The quest for analgesia without adverse $\mathrm{CV}$ effects has been well covered since the worldwide withdrawal of Vioxx in 2004 for its relation to myocardial infarction and stroke. Licofelone (ML3000) is a promising dual inhibitor of COX-1/ COX-2 and 5-LOX enzymes with anti-inflammatory and analgesic properties as well as an improved $\mathrm{CV}$ and gastrointestinal (GI) profile for the treatment of osteoarthritis. Licofelone has good oral bioavailability and reaches peak plasma level 3-4 hours after ingestion. It has a long half-life (approximately 11 hours), with the highest accumulations in liver, lung, kidney, heart, and intestine. ${ }^{20}$

Licofelone is currently in phase 3 trials and has been compared to NSAIDs (Naproxen) and selective COX-2 inhibitors (Celcoxib) in terms of analgesic efficacy and safety profile. A 52-week trial was performed to determine the long-term tolerability and efficacy of licofelone compared with naproxen. This multicenter double-blind trial included patients with symptomatic OA of the knee (as defined by American College of Rheumatology guidelines) who had discontinued NSAID therapy 3-14 days prior to the baseline visit. Patients were randomized to receive licofelone, 100 mg twice daily ( $n=235)$, or naproxen, $500 \mathrm{mg}$ twice daily $(n=229){ }^{21}$

Licofelone treatment was associated with a dose-dependent improvement in WOMAC pain scores from baseline. The efficacy of licofelone $200 \mathrm{mg}$ was similar to that of naproxen during the study, with a trend towards greater efficacy at weeks 26, 39, and 52. Mean changes in WOMAC pain scores, from the baseline value of $63.9 \mathrm{~mm}$, were 27.1, 30.2, and $27.7 \mathrm{~mm}$ for licofelone $100 \mathrm{mg}$, licofelone $200 \mathrm{mg}$, and naproxen, respectively. Safety evaluation of the trial was evaluated by means of GI or CV adverse events. Laboratory parameters and vital signs were also recorded. The incidence of adverse events confirmed the superior long-term tolerability of licofelone 
over naproxen. The patients in the 100 and $200 \mathrm{mg}$ licofelone treatment experienced fewer side effects than those in the naproxen group $(59.2 \%, 56.3 \%$, and $66.7 \%$, respectively). In particular, lower frequencies of peripheral edema and aggravated hypertension were recorded for licofelone compared with naproxen. ${ }^{21}$ These findings of reduced peripheral edema and hypertension suggest the dual inhibition mechanism of licofelone could be free from $\mathrm{CV}$ toxicity in OA patients, an adverse effect otherwise associated with selective COX-2 inhibitors. $^{22}$

To compare the efficacy and tolerability of licofelone with celecoxib in patients with knee OA, a 12-week multicenter, double-blind, parallel-group study was performed. Results indicated that licofelone, $200 \mathrm{mg}$ twice daily, administered to 302 patients was as effective as celecoxib, $200 \mathrm{mg}$ every day, given to 306 patients, with a similar frequency of GI adverse events in both the licofelone group (31.9\%) and the celecoxib group (36.4\%) with significantly fewer incidences of peripheral edema with licofelone as compared to celecoxib. Responders were quantified as those with a $>30 \%$ improvement in WOMAC pain score from baseline. After 12 weeks, responder rates in the licofelone and celecoxib groups were $77.2 \%$ and $77.8 \%$, respectively. ${ }^{21}$

With a heterogeneous patient population, polypharmacy must be considered when pursuing ideal safety profiles in the pharmacologic treatment of OA. The demographic of patients suffering from $\mathrm{OA}$ and seeking analgesia with a low risk burden is growing. To further evaluate the risk burden of licofelone in a patient who is taking more than one medication, the safety profile of licofenole compared with naproxen was explored with the coadministration of aspirin $81 \mathrm{mg}$ daily in a 4-week double-blind, randomized, endoscopy trial. The trial investigated the gastric and duodenal mucosal tolerability, as assessed by endoscopy, of licofelone $200 \mathrm{mg}$ bid and licofelone $400 \mathrm{mg}$ bid compared with naproxen $500 \mathrm{mg}$ bid therapy and placebo over a 4-week period in healthy volunteers. Data revealed that gastroduodenal ulcers of unequivocal depth developed in $20 \%$ of the volunteers receiving naproxen after 4 weeks, while no ulcers were reported in volunteers who received licofelone $200 \mathrm{mg}$ or $400 \mathrm{mg}$. Lanza scores confirmed the significantly superior gastric tolerability of both licofelone doses compared with naproxen, and demonstrated the excellent gastric and duodenal tolerability of licofelone $200 \mathrm{mg}$ and $400 \mathrm{mg}$ compared with placebo. In addition, the tolerability of the treatments did not appear to be effected by positive Helicobacter pylori status. ${ }^{23}$

Naproxcinod (AZD-3582) is the first in a new class of analgesic and anti-inflammatory drugs called COX-inhibiting nitric oxide donators (CINODs). CINODs possess the anti-inflammatory properties of NSAIDs through the balanced inhibition of COX-1 and COX-2 while maintaining an improved GI and CV safety profile. The gastroprotective of CINOD comes from its nitric oxide donating ability. Nitric oxide increases gastric mucus and bicarbonate secretion, improves gastric mucosal blood flow, and inhibits the proinflammatory activities of neutrophils and platelets.

Naproxcinod has completed 3 Phase III clinical studies conducted in the US, Canada, and Europe, which recruited more than 2,700 patients with OA of the knee and hip. Data extrapolated from the most recent phase III clinical trial for Naproxcinod is listed below. Three co-primary endpoints of the study compared the efficacy of naproxcinod $750 \mathrm{mg}$ bid to placebo, in terms of the mean change between baseline and week 13 in the following scores: the WOMAC ${ }^{\mathrm{TM}}$ pain subscale, the WOMAC ${ }^{\mathrm{TM}}$ function subscale and the subject's overall rating of disease status. ${ }^{24}$

In November 2008, a 13-week, double-blind, placebo and naproxen controlled trial in patients with OA of the hip took place. 810 patients were enrolled at 120 clinical centers in the United States, Canada, and Europe. Eligible patients had a diagnosis of primary osteoarthritis of the hip of at least 3 months in duration and were randomized on a 2:2:1 basis to receive naproxcinod $750 \mathrm{mg}$ bid, placebo bid, and naproxen $500 \mathrm{mg}$ bid, respectively. Naproxcinod $750 \mathrm{mg}$ bid showed good overall safety and tolerability. The percentage of patients who experienced one or more GI adverse events were the same for placebo and naproxcinod $750 \mathrm{mg}$ bid at $15.5 \%$, compared with $19.2 \%$ for naproxen $500 \mathrm{mg}$ bid. There was not a single serious CV or serious GI adverse event in the naproxcinod arm during the 13 weeks of the study. In addition, naproxcinod $750 \mathrm{mg}$ bid showed a similar blood pressure profile to placebo, supporting earlier findings suggesting its nondetrimental effect on blood pressure. Naproxinod $750 \mathrm{mg}$ bid showed a clear reduction in systolic and diastolic blood pressure compared with naproxen $500 \mathrm{mg}$ bid at all time points. ${ }^{24}$ In November 2009, NicOx announced that it received a filing communication from the US Food and Drug Administration (FDA) stating that the New Drug Application (NDA) for naproxcinod was accepted for filing. Pending the FDA's approval, naproxinod could be poised for a US launch in late 2010.

Lumiracoxib is a novel COX-2 selective inhibitor developed for the treatment of OA. Lumiracoxib is the only acidic coxib (pKa:4.7), and is more selective for the COX-2 isoform compared with the COX-1 isoform than any other available selective COX-2 inhibitor. ${ }^{25}$ It is structurally distinctive 
from other COX-2 selective drugs by demonstrating a short half-life (4 hours), but sustained pharmacokinetics and pharmacodynamics in target tissue (eg, synovial fluid). These data suggest that lumiracoxib may be associated with reduced systemic exposure, while still reaching sites where COX-2 inhibition is required for pain relief. ${ }^{26}$

The GI and CV safety profile of lumiracoxib was compared with 2 non-steroidal anti-inflammatory drugs, naproxen and ibuprofen, in the Therapeutic Arthritis Research and Gastrointestinal Event Trial (TARGET). TARGET was a 52-week, international, multicenter, randomized, double-blind, parallel-group study that compared $400 \mathrm{mg}$ 4 times daily (for OA recommended dose is $100 \mathrm{mg} /$ day) with ibuprofen $800 \mathrm{mg} 3$ times daily and with naproxen $500 \mathrm{mg}$ twice daily. The study indicated a significantly lower rate of ulcer complications in the group taking lumiracoxib in comparison with those taking nonselective NSAIDs. The lumiracoxib group had significantly fewer ulcer complications compared with those taking NSAIDs. Amongst those taking aspirin, there was no significant difference between lumiracoxib and NSAID patients. ${ }^{14}$

After initially being launched in 2005, lumiracoxib (Prexige) was withdrawn for liver toxicity. Novartis, the maker of lumiracoxib has identified a biomarker in patients with potential hepatic adverse effects and is currently planning resubmission of lumiracoxib with a companion diagnostic biomarker program to the FDA. To certify the molecular biomarker as an effective diagnostic test, Novartis has been working with the pharmacogenic experts at the FDA's Voluntary Exploratory Data Submissions program for their feedback on utilizing the hepatic safety biomarker as a companion test. The resubmission of lumiracoxib could be the "first example" of a molecular diagnostic-based "drug rescue" in the industry. ${ }^{27}$ After the commercial re-launch of lumiracoxib with a widely certified diagnostic test is broadly introduced, Novartis is planning to conduct a prospective observational study to confirm the benefit of genetic testing.

\section{Opiates and their receptors}

The concern over the safety profile of traditional first-line agents such as cyclooxygenase- 2 inhibitors and NSAIDs has limited their use in the pharmacologic management of LBP. In patients who cannot tolerate or have not benefited from the use of traditional first-line agents, conservatively dosed, time-based opioid therapy is becoming a popular alternative. The improved pain control of opioid therapy has allowed the patients suffering from osteoarthritis-induced LBP to become more functionally mobile. This is significant, since inadequate pain control has often left patients immobile, isolated to their home without sunlight which subsequently leaves them deficient in Vitamin D. Among the patients with spinal disorders, The National Medical Expenditure Panel Survey showed a $108 \%$ increase in opioid prescriptions from 1997 through 2004. The combination of increasing use and higher drug prices resulted in a $423 \%$ inflation-adjusted increase in expenditures. ${ }^{28}$

Opioids bind to opioid receptors in the central nervous system and other tissues. Opioid receptors are G-protein coupled receptors acting on GABAergic neurotransmission. There are three principal classes of opioid receptors, $\mathrm{mu}$, kappa, and delta. In addition the opioid receptor-like receptor 1 (ORL1) is an important analgesic target in those who have developed tolerance to mu-opioid agonists.

Clinicians today are finding themselves in a difficult position when it comes to prescribing opiates for their patients suffering from OA due to side effects such as nausea, dependence, hyperalgesia, hypogonadism, respiratory depression, and physical dependence. ${ }^{2}$ The increasing trend to rely upon opioid therapy as an adjunctive therapy for the treatment of a disease process as far reaching as OA, poses the potential to create broad societal impacts. Emerging strategies in utilizing opioids in the pharmacological management of LBP focus on reducing the adverse effects produced by centrally penetrating opiates. Adverse effects of opiate use, such as respiratory depression and constipation, and potential for dependence/abuse are associated with mu-receptor agonists. To circumnavigate around these unwanted effects of mu-opioid receptor agonists, delta opioid receptors, which possess a favorable safety profile, are being targeted. In addition, central mu-opioid receptor agonists are being reformulated to intra-articular and topical forms. ${ }^{14}$

Pharmacology targeting the delta-opioid receptor (DOR) may provide analgesic efficacy without the common side effects of mu-opioid receptor agonists. Emerging DOR biology has indicated that stimulus-dependent (pain, inflammation) trafficking of DOR from cytoplasm to cell surface appears to modulate apparent efficacy of agonists. Thus, delta ligands have low analgesic efficacy in acute pain models but show robust analgesia efficacy in a variety of chronic pain conditions accompanied by inflammation. ${ }^{29}$ Based on these findings the biopharmaceutical company Adolor has developed two delta compounds, ADL5859 and ADL5747. In October 2009, Adolor in collaboration with Pfizer initiated a Phase 2a proof-of-concept study of ADL5859 and ADL5747 in osteoarthritis patients. ${ }^{30}$ Another route for providing opiate analgesia without the side effects of centrally acting opiates is the anti-diarrheal drug Loperamide. In addition to its 
anti-diarrheal capabilities, Loperamide is a mu-opiate receptor agonist that does not penetrate the blood-brain barrier. When mu-opiate receptors are stimulated, they release potassium ions, which cause hyperpolarization of the cell membrane, and makes the neuron less excitable. The release of potassium ions also reduces the calcium ion influx in the terminal end of the neuron, which significantly reduces neurotransmitter release. A neuron with a hyperpolarized cell membrane and diminished ability to release neurotransmitters cannot effectively relay pain signals. The development of topical and intra-articular formulations of loperamide have shown efficacy as a peripherally selective opiate analgesic agent in post operative, inflammatory, and bone cancer pain models. ${ }^{31,32}$

\section{Antiresorptive pharmacology}

Salmon calcitonin (SCT) is an anti-resorptive agent that has been available for over 30 years. SCT is approved for the treatment of post-menopausal osteoporosis, bone associated pain, and metabolic bone disease. SCT is commercially available as an injectable and as a nasal spray. A new oral formulation has been recently developed, and data from the first clinical trials indicate a potential utility not only in osteoporosis but also in osteoarthritis. Recent interest in SCT in the management of $\mathrm{OA}$ is due to its potential chondro-protective capabilities and analgesic effects. While the kinetics of calcitonin-induced pain relief have not been conclusively shown, potential mechanisms include an endorphin-mediated effect through the elevation of endogenous B-endorphin levels, centrally mediated pain relief via central nervous system binding sites, and stimulation of descending spinal serotonergic pathways. ${ }^{33}$

Clinical and pharmacological trial data have been largely focused on the analgesic efficacy of calcitonin as nasal spray (SCT-NS) and as an injectable treatment. Injectable SCT has been shown to be superior to placebo for pain relief in acute vertebral fractures. ${ }^{34}$ The analgesic effects of SCT-NS in vertebral fracture associated pain syndromes have also been shown in a double blind, placebo controlled study. ${ }^{35}$ Overall, the analgesic effects of both nasal spray and injectable preparations are comparable. The oral preparation of SCT has been shown to improve bioavailability and provide higher systemic levels of SCT. In Phase II trials, oral SCT has shown improvements in OA pain. ${ }^{36}$ Currently, Novartis and Nordic Bioscience are evaluating oral SCT (SMC021) in a Phase III trial for OA.

\section{Botulinum toxin}

The mechanism of pain relief provided by botulinum toxin (BoNT) has generated interest after clinical studies indicated that onset of pain relief occurred sooner than muscle spasm relief in studies evaluating BoNT injection for cervical dystonia. ${ }^{37}$ This led to the investigation that pain relief provided by BoNT was separate from its ability to produce reversible muscle paralysis through inhibition of acetylcholine.

Animal studies have indicated multiple mechanisms for the anti-nociceptive properties of botulinum toxin type A (BoNTA). Possible mechanisms include a reduced resting discharge of muscle spindles after injection of BoNTA into skeletal muscles, ${ }^{38}$ which may theoretically diminish central sensitization. In central sensitization, wide-range function neurons of the spinal cord erroneously perceive non-nociceptive input (such as that from muscle spindles) as nociceptive. ${ }^{39}$ In cell cultures and animal studies, BoNTA diminishes the release of pain neurotransmitters such as substance $\mathrm{P}$, calcitonin gene-related peptide, and bradykinin. ${ }^{40}$

In paraspinal compartment syndrome, BoNTA injectioninduced atrophy of bulky paraspinal muscles relieves compression of nerves and vascular structures and reduces ischemic pain. The evidence for BoNTA in the relief of osteoarticular pain has been encouraging. In a double blinded, randomized, placebo controlled study, administration of BoNTA into paraspinal muscles using a novel technique produced significant pain relief in $60 \%$ of patients with chronic refractory back pain. However, this study was limited by a small sample size $(\mathrm{n}=31) .{ }^{41}$ In a larger $(\mathrm{n}=75)$ prospective, randomized open labeled study with a 14 month follow-up, a similar yield of pain relief was seen $(53 \%)$. Among initial responders, 91\% continued to respond over the length of the study. ${ }^{42}$ Currently, the Walter Reed Army Medical Center is holding a Phase IV trial to evaluate the analgesic efficacy of BoNTA in the management of patients suffering from chronic LBP. Botulinum neurotoxin therapy of chronic LBP is an off-label use and is not approved by the US Food and Drug Administration.

\section{Neuropathic pain}

Chronic LBP is highly prevalent in Western societies. Large epidemiological studies show that $20 \%-35 \%$ of patients with back pain suffer from a neuropathic pain component. Presently, chronic lumbar radicular pain is the most common neuropathic pain syndrome. ${ }^{1}$ The revised definition from the International Association for the Study of Pain states neuropathic pain is "pain initiated or caused by a primary lesion or dysfunction in the nervous system". ${ }^{43}$ This definition signifies a shift from the traditional view that neuropathic LBP only relates to radiculopathy. Neuropathic pain is comprised of a complex interplay between peripheral and central mechanisms of pain modulation. 
The complex nature of neuropathic pain has currently left most therapeutic needs unmet. Neuropathic pain can be divided into central and peripheral components. The term 'central' refers to the brain and spinal cord. Central neuropathic pain refers to pain initiated or caused by a primary lesion or dysfunction of the central nervous system. ${ }^{44}$

Anatomically, central pain may originate in any lesion along the neuraxis located in the dorsal horn, the ascending pathways throughout the spinal cord and brain stem, the thalamus, the subcortical white matter, and the cerebral cortex. The most common occurrences of central pain stem from traumatic spinal cord injuries, strokes, and multiple sclerosis. Other causes of central pain include brain tumors, epilepsy, syringomyelia, and spinal cord infractions. ${ }^{45}$

Central neuropathic pain syndromes appear to originate from the reorganization of central somatosensory processing. Central neuropathic pain is associated with neuronal plasticity. Neuronal plasticity refers to the anatomical and neurochemical changes that can occur within the central nervous system. These changes facilitate and maintain a 'pain state' long after the initial injury. This neuronal plasticity or increased excitability reflects a sensitization of neurons within the dorsal horn following peripheral tissue damage. This sensitization is characterized by increased spontaneous activity of the dorsal horn neurons, a decreased threshold and an increased responsivity to afferent input, and cell death in the spinal dorsal horn. ${ }^{46}$

The peripheral component to neuropathic pain pertains to peripheral nerve injury. In peripheral nerve injury, new adrenergic receptors may develop leading to an increased sensitivity to pain. In addition to an increased amount of adrenergic receptors, damaged nerves may possess different depolarization characteristics and dysfunctional sodium channels. These structural aberrations lead to an increased excitability which leads to increased pain transmission.

Pharmacological treatment of neuropathic pain to date is directed towards the symptomatic management of hallmark features such as spontaneous pain, mechanical and cold allodynia, hyperalgesia, and hyperpathia. Recently, an improved molecular-based understanding behind the mechanisms of neuropathic pain has provided better insight and led to development of new therapeutic approaches.

The neuroanatomy of the central and peripheral nervous system and the neuropathic pain spectrum is beyond the scope of this article. Therefore, we will focus on key molecular events and cellular processes in neuropathic pain that provide rationalization for investigational pharmacology.

\section{Pathogenesis}

The neuropathic component to LBP is a not a result of a single pathophysiological mechanism, but the final product of altered peripheral, spinal and supraspinal processing. The initiation of neuropathic LBP may be caused by lesions of nociceptive sprouts within the degenerated disc (local neuropathic), mechanical compression of the nerve root (mechanical neuropathic root pain), or by action of inflammatory mediators (inflammatory neuropathic root pain) originating from the degenerative disc even without any mechanical compression. ${ }^{1}$

\section{lon channel (voltage and ligand gated) blockers}

Hyperexcitability in small and large peripheral sensory nerves acts as an important driving mechanism for neuropathic pain and can account for the initiation and maintenance of central hyperexcitability. ${ }^{43}$ Changes in the expression and activity of several voltage-gated sodium, potassium, and calcium channels have been highlighted after nerve injury. ${ }^{47}$ These changes in the expression, trafficking, and redistribution of ion channels after inflammation or nerve injury are considered to account for unstable oscillations of membrane potential, abnormal firing and the generation of ectopic activity in afferent nerves. ${ }^{48}$ Therefore, the blockage of voltage-gated ion channels is therapeutic in regulating sensory neural excitability.

Lacosamide is an anticonvulsant with neuronal antihyperexcitability properties that is being evaluated as an emerging treatment for neuropathic pain. By blocking voltage-gated sodium channels, Lacosamide has been reported to inconsistently produce significant reduction in pain measures compared with a placebo in clinical trials. ${ }^{47}$ Currently, Lacosamide is in a Phase II trial to assess the continued efficacy and safety of ascending doses in subjects with chronic refractory neuropathic pain.

Another voltage-gated ion channel of interest in the pharmacologic treatment of neuropathic pain is the calcium channel. The influx of calcium ions through voltage-gated calcium channels has an established role in axonal potential formation and neurotransmitter release by primary sensory neurons. Aberrant calcium channel physiology and activity has also become a focus of attention in the search for targets for therapeutic interventions that prevent or alleviate neuropathic pain.

Ziconotide is the first in a new class of non-opioid analgesics known as N-type calcium channel blockers (NCCBs), which target pre-synaptic calcium channels on nerves that ordinarily transmit pain signals. ${ }^{49}$ Intrathecal 
ziconotide infusion was approved by the FDA in 2004 for the management of severe chronic pain in patients who were not effectively managed with systemic analgesics, adjunctive therapies, or intrathecal morphine. Ziconotide is the synthetic equivalent of a naturally occurring conopeptide found in the venom of a marine snail known as Conus magus, a predatory sea creature that attacks its prey with harpoons loaded with a paralytic poison. ${ }^{50}$

Intrathecal ziconotide is regarded as a broad-spectrum analgesic effective as a monotherapy and in conjunction with opioids in the management of neuropathic pain, and to those suffering from intractable pain. In a double-blinded randomized, placebo-controlled trial, patients (169 ziconotide, 86 placebo) with severe chronic nonmalignant pain unresponsive to conventional therapy were treated over a 6-day period in an inpatient hospital setting. The mean percentage change in visual analog scale of pain intensity (VASPI) score from baseline was $31.2 \%$ and $6.0 \%$ for ziconotide- and placebo-treated patients, respectively. ${ }^{51}$ Due to its narrow therapeutic index, ziconotide has been associated with side effects such as postural hypotension, mental status changes and hallucinations. However, significant adverse events are less apt to occur when the drug is slowly titrated gradually over 3 weeks or longer. ${ }^{52}$

The intrathecal administration and adverse effects of ziconotide has limited its clinical utility. Recently, another n-type calcium channel blocker, leconotide (CNSB004) has drawn considerable pharmacologic interest. In animal studies, the anti-hyperalgesic effect of leconotide was comparable to that of ziconotide. When compared with ziconotide, the side effect profile of leconotide (locomotor activity and vascular responses) was clearly better than that of ziconotide. ${ }^{53}$ The maximum no side-effect dose of leconotide caused $51.7 \%$ reversal of hyperalgesia, compared with $0.4 \%$ for the highest no side-effect dose of ziconotide. ${ }^{54}$ In addition, leconotide was effective when administered intravenously, suggesting an effective alternative delivery method for n-type calcium channel blockers other than intrathecal administration. ${ }^{53}$ In the treatment of neuropathic pain, the development of non-opioid analgesics offers new, powerful therapeutic possibilities. These initial findings demonstrate the therapeutic potential of leconotide, a calcium channel blocker with an improved side-effect profile and the ability to be administered through a new route.

In addition to voltage-gated ion channels mentioned above, the large family of transient receptor potential (TRP) vanilboid channels are also pharmacological targets in the treatment of neuropathic pain. Transient receptor potential vanilloid channel 1 (TRPV1), a non-selective cation channel, has garnered significant pharmacological interest. On nociceptive neurons, TRPV1 is gated by capsaicin, noxious heat $\left(>45^{\circ} \mathrm{C}\right)$, acidic $\mathrm{pH}(<5.3)$, and is regulated by a variety of inflammatory mediators (eg, bradykinin and PGE2). ${ }^{47}$ Emerging strategies focus on TRPV1 agonists and antagonists as a molecular focal point in neural sensitization caused by mediators of inflammation and nerve injury. ${ }^{55}$

Capsaicin, a TRPV1-agonist, has been investigated, and reports have been promising. When capsaicin binds to TRPV1, it causes the channel to open below $37^{\circ} \mathrm{C}$. Prolonged activation of these neurons by capsaicin depletes presynaptic substance $\mathrm{P}$, one of the body's neurotransmitters for pain and heat. Capsaicin mimics a burning sensation that leaves the nerves that host a TRPV1 receptor overwhelmed. These nerves are unable to report pain for an extended period of time due to a depletion of neurotransmitters. This depletion of neurotransmitters leads to a reduction in pain sensation. Low dose capsaicin cream $(0.025 \%$ and $0.075 \%)$ has been a moderately efficacious over the counter topical treatment for chronic pain for decades. These low dose capsaicin creams must be applied multiple times a day and have caused a burning pain each time they are applied leading to non compliance.

Transacin (Transdolor, NGX-4010) is a high concentration $(8 \%)$ dermal patch that delivers a therapeutic dose of capsaicin during a 60-minute application. Transaicin (NGX4010) has been well tolerated and efficacious in reducing pain in a number of neuropathic pain conditions including a Phase III study in patients with post-herpetic neuralgia. ${ }^{56}$ Although trials have been limited, due to the difficulty of blinding high-concentration capsaicin, multiple studies both open label and double blinded, suggest that a 1-hour application of NGX-4010 can reduce neuropathic pain in a safe and efficacious manner. Neuropathic pain reductions with NGX-4010 can be maintained with repeated administrations over a 1 -year period. ${ }^{57}$

\section{Cannabinoids}

In 1997, the British Medical Association (BMA), NIH, and American Medical Association published reports concluding that cannabinoids have a role as adjuvant analgesics for pain conditions refractory to standard drugs. ${ }^{47}$

The analgesic success of cannabinoids compounds is centered on targeting 2 receptors within the endocannabinoid system, cannabinoid receptor 1 (CB1) and cannabinoid receptor 2 (CB2). CB1 receptors are widely distributed in the central nervous system and peripheral sensory neurons, 
whereas $\mathrm{CB} 2$ receptors have been found in peripheral tissues including tissues of the immune system and keratinocytes with limited expression in sensory and central nervous system cells. ${ }^{58}$

Studies indicate that cannabinoids such as $\Delta 9$-tetrahydrocannabinol $(\triangle 9$-THC) activate both $\mathrm{CB} 1$ and $\mathrm{CB} 2$ receptors and facilitate cellular inhibitory mechanisms that attenuate the hyperexcitability involved with neruopathic pain. CB1 receptors are responsible for mediating the analgesic effects in the brain and spinal cord, while peripheral CB1 receptors are involved with pain transmission from the peripheral nervous system. ${ }^{59} \mathrm{CB} 2$ receptors are also responsible for pain modulation in the spinal cord.

Multiple clinical trials have shown that treatment utilizing cannabinoid compounds reduce neuropathic pain; however, they also produce adverse effects such as euphoria, dizziness, and sedation. Recent studies have shown selective targeting of peripheral and spinal $\mathrm{CB} 1$ and $\mathrm{CB} 2$ receptors are promising targets for analgesia because they may avoid the psychoactivity of brain $\mathrm{CB} 1$ receptor activation and may allow the administration of doses that produce a greater analgesic effect with reduced psychoactivity. ${ }^{60}$

\section{Cannabinoid receptor pharmacology}

Sativex, an oral form of $(\Delta 9-\mathrm{THC})$ in the preparation of an oralmucosal spray has shown promise as an adjuvant analgesic in the treatment of refractory peripheral neuropathic pain in a Phase II/III, 5-week, double blinded, randomized, placebo-controlled study (324). This study suggests a synergistic interaction between the endocannabinoid and opioid receptor system in the treatment of neuropathic pain. The majority of adverse events associated with Sativex use in this study were categorized as being mild or moderate. Currently, Sativex is an investigational drug in the United States and is being developed as an adjunctive analgesic treatment for patients with advanced cancer whose persistent pain has not been adequately relieved by opioid therapy. Studies have also shown $\mathrm{CB} 2$ selective agonists to modulate pain without central CB1-like side effects. ${ }^{61}$

Although many studies involving cannabinoids in the treatment of neuropathic pain have been promising, there are many challenges that remain. Barriers that need to be overcome prior to utilizing cannabinoid receptors in the treatment of neuropathic pain include an improved definition of therapeutic indications, a better understanding of the therapeutic balance between cannabinoid receptors and opioid receptors, and how to provide effective analgesia while minimizing side effects. Another major barrier to the accepted therapeutic use of cannabinoids in clinical practice is the associated stigma that comes hand in hand with this drug class. ${ }^{62}$

\section{Anti-depressants and B-mimetics}

Despite the close association between chronic pain and depression, we now know that the pain-relieving effect of antidepressants is independent of their mood-elevating properties. ${ }^{63,64}$ The primary mode of action is an interaction with pathways extending through the spinal cord from serotoninergic and noradrenergic structures in the brain stem and midbrain. Other pathways for analgesia provided with antidepressants include actions on opioids, adrenergic, GABA, and N-methyl-D-aspartate receptors.

Tricyclic antidepressants (TCAs) such as amitryptiline, nortryptyline, imipramine, and desipramine are regarded as first-line drugs when treating $\mathrm{LBP}^{65}$ In addition to blocking the reuptake of norepinephrine and serotonin, TCAs block neuronal membrane ion channels and interact with adenosine and NMDA receptors. The utilization of TCAs for neuropathic pain has been limited due to anticholinergic side effects including blurred vision in glaucoma patients, urinary retention, constipation, and dry mouth. Antihistaminergic side effects such as oversedation and weight gain can also preclude its use. ${ }^{66}$

Venlafaxine is a serontonin-norepinephrine reuptake inhibitor (SNRI) and may be considered a suitable alternative to TCAs in relieving neuropathic pain. Venlafaxine does not have anticholinergic and antihistaminergic blocking side effects. ${ }^{67}$ Venlafaxine blocks the reuptake of serotonin at lower doses; when used in higher doses it blocks the reuptake of norepinephrine. When utilized at a high dose there has been concern with Venlafaxine producing hypertension. ${ }^{66}$ Duloxetine, another SNRI commonly used in painful neuropathy, does not have these effects.

Recent studies investigating the mechanism of antidepressants in alleviating neuropathic pain revealed that activation of B-adrenergic receptors (B2-AR) plays a critical role. In a neuropathic pain model, the absence of a B2-AR suppresses the analgesic effects of chronic antidepressant treatment. ${ }^{68,69}$ Preclinical studies have reported that chronic direct stimulation of B2-AR by agonists may relieve neuropathic pain symptoms in a murine neuropathic pain model ${ }^{70-72}$ Drugs that belong to the class of Beta two-adrenergic receptors include albuterol, terbutaline, fenoterol, salmeterol, ritodrine, and isoprenaline among others. These drugs are widely used and have well described adverse effects. Reports on B-mimetics potential as a neuropathic pain treatment are encouraging, and require randomized, controlled, prospective and blinded studies for further evaluation. 


\section{Conclusion}

Unified by the urgency to meet the therapeutic needs of the many that suffer from LBP and provide pain relief with a lower risk burden, the pharmacological landscape for the treatment of chronic LBP is changing as rapidly as any other field in medicine. At the epicenter of this investigational boom is an improved understanding of the biology of pain at a cellular level, safer drug profiles, new routes for drug delivery and novel molecular based therapeutic targets.

The therapeutic challenge of treating LBP stems from treating a pain that is maintained through nociceptive and neuropathic mechanisms, and abnormalities in the excitability of central and peripheral pathways. These factors compounded with a diverse patient population have led to the need for an improved pharmacotherapeutic regimen. This review identified several emerging drugs in clinical Phases II and III, and attempts to provide some insight on how the drug development process is providing viable solutions for managing the complexities of LBP.

As the multimodal approach to managing LBP continues to evolve, our reliance on pharmacotherapy to play a significant role in the treatment algorithm remains constant. The heterogeneity of the LBP population prohibits a single drug from revolutionizing the treatment of LBP, and it is difficult to predict which drug or drugs will establish themselves as the most efficacious. However with the promise of the investigational pharmacology noted in this paper, there is no reason to believe that a larger, safer, and improved therapeutic armamentarium is not forthcoming.

\section{Disclosure}

The authors report no conflicts of interest in this work.

\section{References}

1. Freynhagen R, Baron R. The evaluation of neuropathic components in low back pain. Curr Pain Headache Rep. 2009;13(3):185-190.

2. Deyo R, Mirza S, Turner J, Martin B. Overtreating chronic back pain: time to back off? J Am Board Fam Med. 2009;22(1):62-68.

3. Dagenais S. A systematic review of low back pain cost of illness studies in the united states and internationally. Spine J. 2008;8(1):8-20.

4. Borenstein D. Does osteoarthritis of the lumbar spine cause chronic low back pain? Curr Rheumatol Rep. 2004;6(1):14-19.

5. Altman R. Practical considerations for the pharmacologic management of osteoarthritis. Am J Manag Care. 2009;15(8):236-243.

6. Windsor R, Sullivan K. Lumbosacral disc injuries. eMedicine website. eMedicine, Specialties/Sports Medicine/Spine. Available at http://emedicine.medscape.com/. Accessed on April 9, 2010.

7. Malanga G, Chimes G. Lumbosacral facet syndrome. eMedicine website. eMedicine, Specialties/Sports Medicine/Spine, Available on http:// emedicine.medscape.com. Accessed on April 12, 2010

8. Ashton IK, Ashton BA, Gibson SJ, Polak JM, Jaffray DC. Morphological basis for back pain: the demonstration of nerve fibers and neuropeptides in the lumbar facet joint capsule but not in ligamentum flavum. J Orthop Res. 2005;10(1):72-78.
9. Kalichman L, Hunter D. Lumbar facet joint osteoarthritis: a review. Semin Arthritis Rheum. 2007;37(2):69-80.

10. Pollintine P, Przybyla AS, Dolan P, Adams MA. Neural arch load-bearing in old and degenerated spines. J Biomech. 2007;37(2):197-204.

11. Sowa G, Agarwal S. Cyclic tensile stress exerts a protective effect on intervertebral disc cells. Am J Phys Med Rehabil. 2008;87(7):537-544.

12. Mapp PI, Walsh DA, Bowyer J, Maciewicz RA. Effects of a metalloproteinase inhibitor on osteochondral angiogenesis, chondropathy and pain behavior in a rat model of osteoarthritis. Osteoarthritis Cartilage. 2010;18(4):593-600.

13. Bonnet CS, Walsh DA. Osteoarthritis, angiogenesis and inflammation. Rheumatology. 2005;44(1):7-16.

14. Read S, Dray A. Osteoarthritic pain: a review of current, theoretical and emerging therapeutics. Expert Opin Investig Drugs. 2008;17(5): 619-640.

15. Attur M, Samuels J, Krasnokutsky S, Abramson S. Targeting the synovial tissue for treating osteoarthritis (OA): where is the evidence? Best Pract Res Clin Rheumatol. 2010;24(1):71-79.

16. Funk CD. Prostaglandins and leukotrienes: advances in eicosanoid biology. Science. 2001;294(5448):1871-1875.

17. Martel-Pelletier J, Lajeunesse D, Reboul P, Pelletier J-P. Therapeutic role of dual inhibitors of 5-lox and cox, selective and non-selective non-steroidal anti-inflammatory drugs. Ann Rheum Dis. 2003;62(6): 501-509.

18. Ding C, Cicuttini F. Licofelone (Merckle). IDrugs. 2003;6(8):802-808.

19. de Gaetano G, Donati M, Cerletti C. Prevention of thrombosis and vascular inflammation: benefits and limitations of selective or combined cox-1, cox-2 and 5-lox inhibitors. Trends Pharmacol Sci. 2003;24(5): $245-252$.

20. Kulkarni SK, Singh VP. Licofelone-a novel analgesic and antiinflammatory agent. Curr Top Med Chem. 2007;7(3):251-263.

21. Alvaro-Gracia JM. Licofelone - clinical update on a novel lox/cox inhibitor for the treatment of osteoarthritis. Rheumatology. 2004;43(1): i23-i25.

22. Kulkarni SK, Singh VP. Licofelone: the answer to unmet needs in osteoarthritis therapy? Curr Rheum Rep. 2008;10(1):43-48.

23. Bias P, Buchner A, Klesser B, Laufer S. The gastrointestinal tolerability of the lox/cox inhibitor, licofelone, is similar to placebo and superior to naproxen therapy in healthy volunteers: results from a randomized, controlled trial. Am J Gastroenterol. 2004;99:611-618.

24. Third pivotal phase 3 study for nicox' naproxcinod shows positive efficacy, safety and blood pressure results. Available from: http://www. medicalnewstoday.com. Accessed on Mar 2, 2010.

25. Bannwarth B, Bérenbaum F. Lumiracoxib in the management of osteoarthritis and acute pain. Expert Opin Pharmacother. 2007;8(10): 1551-1564.

26. Rordorf C, Choi L, Marshall P, Mangold J. Clinical pharmacology of lumiracoxib: a selective cyclo-oxygenase-2 inhibitor. Clin Pharmacokinet. 2005;44(12):1247-1266.

27. Toner B, Ray T. Novartis adds companion dx to lumiracoxib resubmission in Europe. Pharmacogenomics Reporter. Available from: http://www.genomeweb.com. Accessed on Mar 15, 2010.

28. Martin B, Deyo R, Mirza S, Turner J. Expenditures and health status among adults with back and neck problems. JAMA. 2008;299(6):656-664.

29. Cahill C, Morinville A, Lee M-C. Prolonged morphine treatment targets delta opioid receptors to neuronal plasma membranes and enhances delta-mediated antinociception. J Neurosci. 2001;21(19):7598-7607.

30. Delta opioid receptor agonist program. Available from: http://www. adolor.com. Accessed on May 20, 2010.

31. Menéndez L, Lastra A, Meana A, Hidalgo A, Baamonde A. Analgesic effects of loperamide in bone cancer pain in mice. Pharmacol Biochem Behav. 2005;81(1):114-121.

32. Whiteside GT, Boulet JM, Walker K. The role of central and peripheral $\mu$ opioid receptors in inflammatory pain and edema: a study using morphine and dipoa ([8-(3,3-diphenyl-propyl)-4-oxo-1-phenyl-1,3,8triaza-spiro[4.5]dec-3-yl]-acetic acid). J Pharmacol Exp Ther. 2005; 314(5):1234-1240. 
33. Silverman SL, Azria M. The analgesic role of calcitonin following osteoporotic fracture. Osteoporos Int. 2002;13(11):858-867.

34. Lyritis GP, Tsakalakos N, Magiasis B, Karachalios T. Analgesic effect of salmon calcitonin in osteoporotic vertebral fractures: a double-blind placebo-controlled clinical study. Calcif Tissue Int. 1991;49(6): 369-372.

35. Lyritis GP, Paspati I, Karachalios T, Ioakimidis D. Pain relief from nasal salmon calcitonin in osteoporotic vertebral crush fractures. a double blind, placebo-controlled clinical study. Acta Orthop Scand Suppl. 1997;275:112-114.

36. Manicourt D-H, Azria M, Mindeholm L, Thonar E. Oral salmon calcitonin reduces lequesne's algofunctional index scores and decreases urinary and serum levels of biomarkers of joint metabolism in knee osteoarthritis. Arthritis Rheum. 2006;54(10):3205-3211.

37. Jankovic J, Schwartz K. Botulinum toxin injections for cervical dystonia. Neurology. 1990;40:277-280.

38. Filippi J, Errico K, Santarelli R, Bagolini B. Botulinum a toxin effects on rat jaw muscle spindles. Acta Oto-laryngol. 1993;113(3):400-404.

39. Jabbari B. Treatment of chronic low back pain with botulinum neurotoxins. Curr Pain Headache Rep. 2007;11(5):352-358.

40. Foster L, Clapp L, Erickson M, Jabbari B. Botulinum toxin a and chronic low back pain. Neurology. 2001;56:1290-1293.

41. Jabbari B, Ney J, Sichani A. Treatment of refractory, chronic low back pain with botulinum toxin a: a prospective open label study with 14 months follow up. Pain Med. 2006;7:260-264.

42. Aoki KR. Treatment of chronic low back pain with botulinum neurotoxins. Headache J Head Face Pain. 2003;43(1):9-15.

43. Woolf C, Salter M. Neuronal plasticity: increasing the gain in pain Science. 2000;288(5472):1765-1768.

44. Merskey H, Bogduk N editors. Classification of Chronic Pain: Descriptions of Chronic Pain Syndromes and Definitions of Pain Terms. 2nd ed. Seattle: IASP Press; 1994.

45. Pappagallo M editor. The Neurological Basis of Pain. 1st ed. New York: McGraw-Hill; 2004

46. Richeimer S. Understanding neuropathic pain. Spine Universe website. Available from: http://www.spineuniverse.com/conditions/ understanding-neuropathic-pain. Accessed on Jun 2, 2010.

47. Dray A. Neuropathic pain: emerging treatments. Br J Anaesth. 2008; 101(1):48-58.

48. Devor M. Sodium channels and mechanisms of neuropathic pain. J Pain. 2005; 7(1):S3-S12.

49. Mathur M. Ziconotide: a new pharmacological class of drugs for the management of pain. Semin Anesth. 2000;19:67-75.

50. Olivera B, Gray WR, Zeikus R, McIntosh JM. Peptide neurotoxins from fish hunting cone snails. Science. 1985;230(4732):1338-1343.

51. Wallace M, Charapata S, Fisher R. Intrathecal ziconotide in the treatment of chronic nonmalignant pain: a randomized, double-blind, placebocontrolled clinical trial. Neuromodulation. 2006;9(2):75-86.

52. Smith H, Deer T. Safety and efficacy of intrathecal ziconotide in the management of severe chronic pain. Ther Clin Risk Manag. 2009;5: 521-534.

53. Mao J. N-type calcium channel blocker for pain treatment. Pain Med. 2010;11(2):262-273

54. Kolosov A, Goodchild C, Cooke I. Cnsb004 (leconotide) causes antihyperalgesia without side effects when given intravenously: a comparison with ziconotide in a rat model of diabetic neuropathic pain. Pain Med. 2009;11(2):262-273.

Journal of Pain Research

\section{Publish your work in this journal}

The Journal of Pain Research is an international, peer-reviewed, open access, online journal that welcomes laboratory and clinical findings in the fields of pain research and the prevention and management of pain. Original research, reviews, symposium reports, hypothesis formation and commentaries are all considered for publication.
55. Ramsey J, Delling M, Clapham D. An introduction to TRP channels. Annu Rev Physiol. 2006;68:619-647.

56. Szallasi A, Blumberg P. (capsaicin) receptors and mechanisms. Pharmacol Rev. 1999;51(2):159-212.

57. Backonja MM, Malan P, Vanhove G, Tobias J. Ngx-4010, a highconcentration capsaicin patch, for the treatment of postherpetic neuralgia: a randomized, double-blind, controlled study with an open-label extension. Pain Med. 2010;11(4):600-608.

58. Garcia-Martinez C, Humet M, Planells-Casas R. Attenuation of thermal nociception and hyperalgesia by VR1 blockers. Proc Natl Acad Sci U S A. 2002;99(4):2374-2379.

59. Agarwal N, Pacher P, Tegeder I, Amaya F. Cannabinoids mediate analgesia largely via peripheral type 1 cannabinoid receptors in nociceptors. Nat Neurosci. 2007;10:870-879.

60. Gutierrez T, Farthing JN, Zvonok AM, Makriyannis A. Activation of peripheral cannabinoid $\mathrm{CB} 1$ and $\mathrm{CB} 2$ receptors suppresses the maintenance of inflammatory nociception: a comparative analysis. $\mathrm{Br}$ J Pharmacol. 2007;150(2):153-163.

61. LaBuda C, Koblish M, Little P. Cannabinoid CB2 receptor agonist activity in the hindpaw incisionmodel of postoperative pain. Eur $J$ Pharmacol. 2005;527(1):172-174.

62. Turcotte T, Le Dorze JN, Esfahani AM, Frost A. Examining the roles of cannabinoids in pain and other therapeutic indications: a review. Expert Opin Pharmacother. 2010;11(1):17-31.

63. Woodforde JM, Dwyer B, McEwen BW, DeWilde FW, Bleasel K. Treatment of post-herpetic neuralgia. Med J Aust. 1965;(2): 869-872.

64. Max MB, Culnane M, Schafer SC, Gracely RH, Walther DJ. Amitryptyline relieves diabetic neuropathy pain in patients with normal or depressed mood. Neurology. 1987;(37):589-596.

65. Salerno SM, Browning R, Jackson JL. The Effect of antidepressant treatment on chronic back pain: a meta-analysis. Arch Intern Med. 2002;(162):19-24.

66. Chan HN, Fam J, Ng BY. Use of antidepressants in the treatment of chronic pain. Ann Acad Med Singapore. 2009;(38):974-979.

67. Vranken J. Mechanisms and treatment of neuropathic pain. Cent Nerv Syst Agents Med Chem. 2009;(9):71-78.

68. Yalcin I, Tessier LH, Petit-Demouliere N, Doridot S, Hein L. Beta 2 -adrenoreceptors are essential for desipramine, venlafaxine or reboxetine action in neuropathic pain. Neurobiol Dis. 2009;(33): 386-394.

69. Yalcin I, Choucair-Jaafar N, Benbouzid M, Tessier LH, Muller A. Beta (2)-adrenoreceptors are critical for antidepressant treatment of neuropathic pain. Ann Neurol. 2009;(65):218-225.

70. Barrot M, Yalcin I, Choucair-Jaafar N, Benbouzid M, Freund-Mercer MJ. From antidepressant drugs to beta-mimetics: preclinical insights on potential new treatments for neuropathic pain. Recent Pat CNS Drug Discov. 2009;(4):182-189.

71. Choucair-Jaafar N, Yalcin I, Rodeau J, Waltisperger E, Freund-Mercier M. B2-adrenoceptor agonists alleviate neuropathic allodynia in mice after chronic treatment. Br J Pharmacol. 2009;(158):1683-1694.

72. Yalcin I, Tessier LH, Petit-Demouliere N, Waltisperger E, Hein L. Chronic treatment with agonists of beta(2)-adrenergic receptors in neuropathic pain. Exp Neurol. 2010;(221):115-121.

\section{Dovepress}

The manuscript management system is completely online and includes a very quick and fair peer-review system, which is all easy to use. Visit http://www.dovepress.com/testimonials.php to read real quotes from published authors. 